\title{
Pattern-Based Machine Translation
}

\author{
Koichi Takeda \\ Tokyo Research Laboratory, IBM Research \\ 1623-14 Shimotsuruma, Yamalo, Kanagawa 242, Japan \\ Phone: 81-462-73-4569, 81-462-73-7413 (F^X) \\ takedadrl.vnet. ibm. com
}

\begin{abstract}
In this paper, we describe a "pattien-based" mathine transtation (M'l') approath that we followed in clesigning a. persotial tool for users who havo access to large volumes of text in languages other than their own, such as WWW pages. Some of the critical issues involved in the design of such a tool indude easy customization for diverse domains, the officiency of the translation algorithm, and scalability (inceremental improvement in translation quality through user interaction). We also describo how our patterns fit into the context-free parsing and generation algorithms, and how we implemented a prototype tool.
\end{abstract}

\section{Introduction}

It would be difficult for anyone to clispute the idea that the World-Wirle Web (WWW) has been the most phenomenal invention of the last decade in the computing envimonment. It has suddenly opened 11p as winclow to vast amounts of data on the Internet. Unfortunately for those who are not native English spoakers, textual data. are more often than not written in a foreign language.

$\Lambda$ dozen or so machine translation (MT) tools bave recently been prot on the market, to make such textual datia more accessible, but novice P' ${ }^{\prime}$ users will be simply amazed at the moagerness of their reward for the effort of builcling a so-called "11ser dictionary."

'The main reasons for this problem are:

1. Most M'T' systems do not employ a powerful "lexicalist" formalism.

2. Most M'T systems can be customized only by adding a. user dictionary.

Therefore, user's can neither givo preferences on individual prepositional-phrase attachments (e.g., to obtain information from a server nor define translations of specific verb-object pairs (e.g, to take advantage of something).

Powerful grammar formalisms and lexical-semantics formalisms have been known for years (see l. $\mathrm{H}(\mathrm{G}(\mathrm{Ka})$ ) lan and Bresnan, 1982), MPSG(Pollard and Sag, 1987), and Generative Lexicon(P1stojovsky, 1991), for example), but practical implementation of an M'l' system has yet to taxkle the computational complexity of parsing algorithms for these formalisms and the workload of building a large-scale lexicon.

Example based MT'Sato and Nagao, 1990; Sumita and lida, 1991) and statistical M'T(Brown et al., 1993) are both promising approakthes that generally demonstrate incremental improvement in translation axecuracy as the quality of examples or training clata grows. It is, however an open question whether these approaches alone can be nsed to create a full-fledged M'T system; that is, it is mocetain whether such a system can be? used for various domains without showing sovere degradation in translation acceracy, or if it has to be fed by a. reasonably large set of examples or training clata for ach now domain.

'WA-based M'T'( $\Lambda$ beillé ot al., 1990$)^{\prime}$ and pattern. based translation(Maruyama, 1993) share many impor-

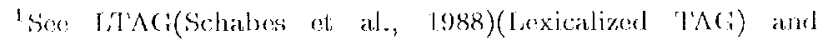

tant properties for sucecessful implementation in practical M'T systems, namely:

- 'The cxistence of a polynomial-time parsing algorithm

- A capability for clescribing a larger domain of locality

- Symchronization of the source and target language structures

In this paper, we show that there exists an attractive way of crossing these approaches, which we call patternbased MT." lin the following two sections, we introduce a class of translation "patterns" based on ContextFreo Grammar (CFG), and a parsing algorithm with $O\left(|G|^{2} n^{4}\right)$ worst-case time complexity. Furthermore, we show that on framework can be extended to incorporate examplo-based MT and a powerful learning mochanism.

\section{Translation Patterns}

A translation pattern is defined as a pair of CHC rules, and zero or more syntactic head and link constraints for nonterminal symbols. For example, the English-French translation pattern ${ }^{3}$

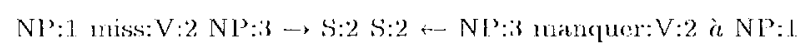

essentially describes a synchronized ${ }^{4}$ pair consisting of a left-hand-side Finglish CFG rule (called a source rule)

$\mathrm{NP} V \mathrm{NP}^{3} \rightarrow \mathrm{S}$

and a right-hand-sicle French CFG rule (called a target rule)

$\mathrm{S} \leftarrow \cdot \mathrm{NP} V$ à NP

accompanied by the following constraints:

1. Head constraints: "The nonterminal symbol $V$ in the source rule must have the verb "miss" as a syntactic head. "The symbol $V$ in the target rule must have the verb "mancuer" as a syntactic head. 'Tho head of symbols in the source (target) rule is identical to the head of symbol $V$ in the source (target) rule, as they are co-indexed. Head constraints can be specified in either or both sides of the pattems.

2. Link constraints: Nonterminal symbols in source and target $\mathrm{CHC}$ rules are linked if they are given the same index ": $i$ ". Thus, the first NP" (NI":1) in the sontec rule corresponds to the second $\mathrm{VI}^{\prime}(\mathrm{NP}: 1)$ in the target rule, the $V_{s}$ in both rules correspond to each other, and the second $\mathrm{NP}\left(\mathrm{NI}^{3}: 3\right)$ in the source rule corresponds to the first NP' (NT:3) in the target rule.

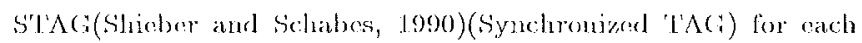
member of the TAC: (T're Adjoining (Prammar) family.

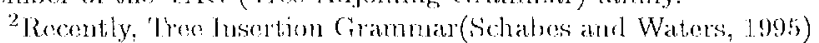
has ben introduced to show a siunilar possibility. Onr approach, bowever, is more inelined toward the cofer fomalism.

"And its inflectional variduts - we will discenss ingreement issucs later, in the "lixtended lormalism" section.

the meaning of the word "synelhonized" here is exactily the sinue as in S'LAci(shober and sohabes, 1990). 
The source and target rules, that is, the CFG rules with no constraints, are called the $C F G$ skeleton of the patterns. The notion of a syntactic head is similar to that used in unification grammars, although the heads in our patterns are simply encoded as character strings rather than as complex feature structures. A head is typically introduced ${ }^{5}$ in preterminal rules such as

$$
\text { leave } \rightarrow \mathrm{V} \mathrm{V} \leftarrow \text { partir }
$$

where two verbs, "lcave" and "partir," are associated with the heads of the nonterminal symbol V. This is equivalently expressed as

$$
\text { leave: } 1 \rightarrow \mathrm{V}: 1 \mathrm{~V}: 1 \leftarrow \text { partir: } 1
$$

which is physically implemented as an entry of a lexicon.

A set $\mathrm{T}$ of translation patterns is said to accept an input $s$ iff there is a derivation sequence $Q$ for $s$ using the source CFG skeletons of T, and every head constraint assoriated with the CFG skeletons in Q is satisfied. Similarly, $T$ is said to translute $s$ iff there is a synchronized clerivation sequence $Q$ for $s$ such that ' $T$ ' accepts $s$, and every head and link constraint associated with the source and target $\mathrm{CHG}^{2}$ skeletons in $\mathrm{Q}$ is satisfied. The derivation $Q$ then produces a translation $t$ as the resulting sequence of terminal symbols included in the target $\mathrm{CFG}$ skeletons in $Q$. Translation of an input string $s$ essentially consists of the following thrce steps:

- Parsing $s$ by using the source CFG skeletons

- Propagating link constraints from source to target CFG skeletons to build a target CFG derivation sequences

- Generating $t$ from the target CFG derivation sequence

The third step is trivial as in the case of ST $\Lambda$ G translation.

Some immediate results follow from the above definitions. (Takeda, 1996)

1. Let a CFG grammar $\mathrm{C}$ be a set of source $\mathrm{CFG}$ skeletons in T. Then, ' $T$ accepts a context-free language (CFL), denoted by $L(T)$, such that $L(T) \subseteq L(G)$.

2. Let a CFG grammar $H$ be a subset of source $\mathrm{CFG}$ skoletons in $T$ such that a source CFG skeleton $k$ is in II iff $k$ has no head constraints associated with it. Then, $H$ accepts a subset $L(H)$ of language $L(T)$.

3. $L\left(T^{\prime}\right)$ is a proper subset of $L(G)$ if, for example, there exists a pattern $p(\in \mathrm{T})$ with a source $C F G$ rule $X \rightarrow X_{1} \cdots X_{k}$ such that ${ }^{\mathfrak{G}}$

(a) $p$ has a head constraint $h: X$ for some nonterminal symbol $X_{i}(i=1,2, \ldots, k)$.

(b) Thas a derivation sequence $X \rightarrow \cdots \rightarrow w$ such that $\mathrm{X}$ is associated with a head $g(h \neq g)$, and $\mathrm{T}$ has no sequence of nonterminal symbols $Y_{1} \ldots Y_{l}$ that derives exactly the same set of strings as $\mathrm{X}$ doos.

\footnotetext{
${ }^{5}$ A nonterminal symbol $X$ in a source or target CFG rule $X \rightarrow$ $X_{1} \ldots X_{k}$ can only be constrained to have one of the lieads in the RHS $X_{1} \cdots X_{k}$. Thus, monotonicity of hoad constraints holds throughout the parsing process.

"This is not a necossary condition for $L\left(I^{\prime}\right) \subset L\left(G^{\prime}\right)$. It is provable that for any set $\mathrm{T}$ of patterns, there exists a (weakly) equivalent CFG grammar $F$, with possibly exponentially more grammar rules, such that $L(T)=L\left(F^{2}\right)$. $\Lambda$ decision problem of Lwo Clis, $L(T) \subset L(G)$, is solvable iff $L(F)=L(G)$. 'l.his includes an undecidable problem, $I(F)=\Sigma^{-k}$. Therefore, we can conclude that $L(T) \subset L(G)$ is undecidable. Similar discussions can be found in the literature on Generalized Phrase Structure Grammar(Gazdar et al., 1985).
}

Although our "patterns" have no more descriptive power than CFG, they can provide considerably better descriptions of the domain of locality than ordinary $\mathrm{CHG}$ rules. For example,

be:V:1 year: NP:2 old $\rightarrow \mathrm{VP}^{r}: 1$ VP: $1 \leftarrow$ avoir: $\mathrm{V}: 1$ an: NI':2

can handle such NP pairs as "one year" and "un an," and "more than two years" and "plus que deux ans," which would have to be covered by a large number of plain CFG rules. TAGs, on the other hand, are known to be "mildly context-sensitive" grammars, and they can capture a wider range of syntactic dependencies, such as cross-serial dependencies. The computational complexity of parsing for TAGs, however, is $O\left(|G| n^{6}\right)$, which is far greater than that of $\mathrm{CFG}$ parsing. Moreover, defining a new STAG rule is not as easy for the nsers as just adding an entry into a dictionary, becanse each $\mathrm{ST \Lambda G}$ mule has to be specified as a pair of syntactic tree structures. Our patterns, on the other hand, can be specified as easily as

$$
\begin{aligned}
& \text { to leave } * \text { de quitter } * \\
& \text { to be year:* old }=\text { d'avoir an:* }
\end{aligned}
$$

by the users. Hore, the wildcard "*" stands for an NP by default. The prepositions "to" and "de" are merely used to specify that these pattorns are for VPs, and they are removed when compiled into internal forms so that these patterns are applicable to finite as well as infinite forms. Similarly, "to be" is used to show that the phrase is a be-verb and its complement. The wildcards can be constrained with a head, as in "year:*" and "an:*". In addi tion, they can be associated with an explicit nonterminal symbol such as "V:*" or "ADJP:*" (e.g., "leave:V:*"). By defining a few such notations, these patterns can bo successfully converted into the formal representations defined above. 'The notations are so simple that even a novice PC user should have no trouble in writing our patterns, as if he or she were making a vocabulary list for English or French exams.

\section{Pattern-Based Translation Algorithm}

$\Lambda$ parsing algorithm for translation patterns can be any of the known CFG parsing algorithms, including CKY and Earley algorithms. It should be first noted, however, that CFG could produce exponontially ambignous parses for some input, in which caso we can only apply heuristic: or stochastic measurement to select the most promising parse.

It is known that an Warley-based parsing algorithm can be made to run in $O\left(|G| K n^{3}\right)$ rather than $O\left(|G|^{2} n^{3}\right)$,(Maruyama, 1993; Graham et al., 1980) where $\mathrm{K}$ is the number of distinct nonterminal symbols in the grammar G. We can expect a very efficient parser for our patterns. 'The input string can also be scanned to reduce the number of relevant grammar rules before parsing." The combined process is also known as offlineparsing in LTAG.

Fandling ambiguous parses is a difficult task. The basic strategy for choosing a candidate parse cluring Jarleybased parsing is as follows:

1. Prefer a pattern $p$ with a source CHG skeleton $X \rightarrow$ $X_{1} \cdots X_{k}$ over any other pattern $q$ such that the source CFG skeleton of $q$ is $X \rightarrow X_{1} \ldots X_{k}$, and such that $X_{i}$ in $p$ has a head constraint $h$ if $q$ has $h: X_{i}(i=1, \ldots, k)$. The pattern $p$ is said to bo more specific than $\%$. This relation is similar to a subsumption relationship(Pollard and Sag, 1987).

\footnotetext{
${ }^{7}$ Schabes and Waters(Schabes and Waters, 1095) also discuss sevoral techniques for optimining parsing algorithus.

8 such scamning is essential for some languages with no explicit word boundaries (such as Japanose and Chinese).
} 
2. Prefor a pattern $p$ with a source CFG skeleton over one with fewer terminal symbols than $p$.

3. Prefer a pattern $p$ that does not violate any heak constraint over one that violates a head constraint.

4. Prefer the shortest alerivation secuence for each input substring. A pattern for a larger domain of locality tends to give a shorter derivation sefuence.

Thus, our strategy favors lexicalized (or head constrained) and collocational patterns, which is exactly what we are going to achieve with pattern-based M'T. Selection of patterns in the derivation seduence accomparnes the construction of a target derivation secpuence. link constraintss are propagated from source to target derivation trees. This is basically a bottom-11p proce.dure.

Since the number $M$ of distinct pairs $\langle X, w\rangle$, for a norlterminal symbol (or a chart) $X$ and a sibbsefuence $w$ of input string $s$, is bonnded by $k n^{2}$, there are at most Kn: possible triples $\langle X, \mathrm{w}, \mathrm{h}\rangle$, such that $h$ is a hearl of $X$. T'hus, we can compute the m-best choice of trarshation canclidates in $O\left(|T| K n^{4}\right)$ time. Here, $K$ is the number of distinct nonterminal symbols in $\mathrm{T}$, and $n$ is the size of the input string.

The reader should note critical differences between lex icalized grammar rules (in the sense of I'JA $(\mathrm{Y}$ ) and translation patterns when they are used for M'T

Firstly, a pattern is not nocessasily lexicalized. An conomical way of organizing translation patterns is to include non-lexicalized patterns as "lefault" translation rules. For example, the patteru

$$
\mathrm{V}: 1 \mathrm{NI}: 2 \rightarrow \mathrm{VI}: 1 \mathrm{VP}: 1<-\mathrm{V}: 1 \mathrm{NP}: 2
$$

is used as a clefault translation of "verb - - direct objoct" expressions, but

resemble: V:1 NP:2 $\rightarrow$ VP:1 VP:1 - resembler:V:1 is N1:2

is always preferred over the default rule because of our preference strategy. Similarly, the pattern

please VP:1 $\rightarrow$ VP:1 VP:I i- VP'I, s'il vous plait

should be preforred over a lexicalized pattern, if any,

NDVP:1 xxx:VP::2 $\rightarrow$ VP:2 VI:2 - AIVPP:1 yyy:VI::2

Serondly, lexicalization might ensiderably increase the size of STAG grammars (in particular, compositional grammar rules such as $A D . J P N^{\prime} \rightarrow N P$ ') when a large number of lexical items are associated with them. Sinco it is not unusual for a noun in a source language to have several counterparts in a target language, the number of trec-pairs in STAG would grow much larger than that of somrce LTAG troes. Nlthough in LTAG the grammar rules are differentiated from their physical objects ("parser rules"), and "structure sharing" (Vijay-Shanker and Schabos, 1992) is proposed, this ambiguity remains in the parser rules, too.

Thirdly, a translation pattern can omit the tree structure of a collocation, leaving it as just a secpuence of torminal symbols. For example,

Soo you later, $\mathrm{Nl}^{\prime} \mathrm{L} \rightarrow \mathrm{S} S \leftarrow$ Au revoir, Nl':l

is perfectly acceptable as a translation pattern.

\section{Extended Formalism}

Syntactic dependencies in natural language sentences are so subtle that many powerful grammar formalisms have boen proposed to account for them. The adequacy of CFG for describing natural language syntax has long been questioned, and unification grammars, among others, have been used to build a precise theory of the computational aspects of syntactic dependencies, which are described by the notion of unification and by feature structures.

Translation patterns can also be extended by means of unification and feature structures. Such extensions must bo carefully applied so that they do not sacrifice the of ficiency of parsing and generation algorithms. Shieber and Schabes briefty riscuss the issue(Shicher and Schabes, 1990$)$. Wo can also extend translation patterns as follows:

Lach nonterminal node in a pattern can be as sociated with a fixed-length vector of binary features.

This will onable us to specify such syntactic dependencios as agreement and subcategorization in patterns. Unifi(aition of hinary features, however, is much simples: moification of a feature-value pais succeeds only when the pair is oither $(0,0)$ or $(1,1)$. Since the feature vector has a fixed length, nnification of two featume vectors is performed in a constant time. For example, the patterns

V:1:+TRANS NP:2 $\rightarrow$ VP:1 VP:J - V:1:+'TRANS NI::2

$V: 1:+$ INTRANS $\rightarrow$ VP: 1 VP:1 $\leftarrow$ V:1:AIN'TRANS

are unifiable with transitive and intransitive verbs, ro spectively. We can also distinguish local and head features, as postulated in IIJ'SG. Verb subcategorization is then encoled an

VP:L:-'TR $\triangle$ NS-OB.I NP:2 $\rightarrow$ VP:1:+0I3.T

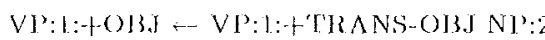

where "-013.J" is a local feature for head VI's in l,HSs while "+OB.J" is a head feature for VI's in the licliss. Unification of a head feature with +oBJ succeeds when it is not bound.

Another extension is to associate weights with patterns. It is then possible to rank the matching patterns according to a linear ordering of the weights rather than the pairwise partial ordering of patterns described in the previous section. Numeric: weights for patterns are extromely useful as a means of assigning higher priorities to user-clefined patterns.

The final extension of translation patterns is integrat tion of examples, or bilingual corpore, into our framework. It consists of the following steps. [eet 'T' be a set of translation patterns, $B$ a bilingual corpus, and $(s, t)$ a pair of source and target sentences.

1. If ' $T$ can transtate $s$ into $t$, do nothing.

2. If ' $T$ can translate $s$ into $t^{\prime}\left(t \neq t^{\prime}\right)$, clo the following:

(a) If there is a paired derivation sequence $Q$ of $\langle s, t\rangle$ in $\mathrm{T}$, create a new pattern $p^{\prime}$ for a pattern $y$ used in $Q$ such that every nonterminal symbol $X$ in $p$ with no head constraint is associated with $h: X$ in $q$, where the head $h$ is instantiated in $X$ of $p$. Adel $p^{\prime}$ to ' $\mathrm{T}$ ' if it is not al ready there.

(b) If there is no such paired derivation sequence, add the pair to $T\langle s, t\rangle$ as a translation pattern.

3. If T' cannot translate $s$, add the pair $\langle s, t\rangle$ to $\mathrm{T}$ as a translation pattern.

The simplest way of integrating the corpus $B$ into ' $T$ ' is just to consider the sentence pair $\langle s, t\rangle$ as a translation pattern. Some additional steps are necessary to achiove higher M'T accuracy for a slightly wider range of sentences than those included in 13. However, the degree of improvement in MT accuracy that can be achicved with this learning mechanism is open to question, since the adclition of translation patterns does not necossarily guarantee a monotonic improvenent in M'T accuracy. 


\section{Implementation}

Our experimental implementation of a pattern-based MT system consists of about 500 default-translation patterns, about 2400 idiomatic and collocational patterns, and about 60,000 lexical items for English-to-Japanese translation. A sample run of the prototype system is shown in Figure 1. It shows one of the derivation sequences for the input sentence

Tohn should hear from Mary about the news if he returns home.

Each line in the derivation sequence shows an Fnglish source CFG rule of a pattern used for the derivation. For example, the first line

[( $\left.\begin{array}{ll}0 & 13\end{array}\right) \mathrm{S}: 1: /$ eFIN , ePRES, eSUBJ , eAUX/

$\rightarrow$ S1:1: +eFIN PUNCT:2

in the dorivation sequence shows that two nonterminal symbols, $S 1$ and PUNCT, form a sentence $S$, that $S$ is co-indexed with $\mathrm{S} 1$ and that $\mathrm{S} 1$ must have a finite form feature teFTN. The current instance of $S$ has four foatures - finite, present (cPRES), with-subject (eS(JB.J), and with-auriliary-verb (eAUX) -.. and it spans the word positions 0 to $13 .^{9}$ We can also find several head-constrained patterns there. For example,

[(10 12) VP: 1:/eFIN, e3SG, ePRES, eDBJ, eSAT/ $\rightarrow$

VP"return" $: 1:-$ eOBJ NP"home" $: 2:$ teCAUS

is a pattern for translating "roturn: $V$ home: $N P$ ". Tho clefault $\mathrm{V}+\mathrm{NP}$ translation pattern will assign a wrong Japanese case marker for this phrase.

Our prototype took about 9 sec (clapsed time) to translate this input sentence and produce sevon alternative translations. The derivation shown in the figure was the first (i.e., the best), and generates a correct translation. 'Therefore, collocational patterns and defanlt patterns have been appropriately combined under onr preference strategy.

\section{Conclusions and Future Work}

In this paper, we have proposed a pattern-based M'. system that satisfies three essential requirements of the current market: efficiency, scaldbility, and ease-of-use. We are aware that CFG-based patterns are less adequate for describing syntactic dependencies than linguistically motivated grammar formalisms such as T $\Lambda$ Gs and TIPSC. To achieve the best possible average runtime and accuracy, perhaps our pattern-based system should be combined with more powerfinl grammar formalisms. We believe that the theory and implementation of patternbased M'T will contribute to the realization of computational linguistic theories. A corpus integration method to verify efficiency of the grammar accuisition has yot to be implemented.

Some of the assumptions on patterts should be reexamined when wo extend the definition of patterns. The notion of Thead constraints may have to be extended into that of a set nombership constraint if we need to handle: coordinated structures. Some light-verb phrases cannot be correctly translated withont "exchanging" several feature values between the verb and its object. A similar problem has been found in be-verb phrases.

Oother fatiures include nominative and causative cases, 3rotporson-singular forms, and capitalized words. 'l'wo fintures, "*ARCS" and "*ARCVV" are special ones for remesenting subject vorb agreoment without splititing a pattern into an equivalont set of several patitens for a specific type of agreenent. 'This sourece derivation sequence is actually accompanied by its Japanese counterpart, which was omithed due to the space limitation.
$>$ John should hear from Mary about the news if he returns home.

$\left[\left(\begin{array}{l}0 \\ 0\end{array} 13\right)\right.$ S $: 1: /$ eFIN, ePRES, eSUBJ, eAUX $\rightarrow S 1: 1:+e F I N$ PUNCT $: 2$ $\left[\left(\begin{array}{ll}0 & 12\end{array}\right) \quad S 1: 2: / \mathrm{eFIN}\right.$, ePRES, eSUB $]$, eAUX $/ \rightarrow$

NP: $1: * e A G R S+e N O M I$ VP: $2: *$ eAGRV+eFIN-eSUB

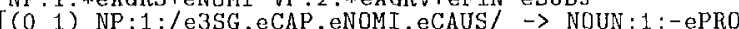

$\left[\left(\begin{array}{ll}0 & 1\end{array}\right)\right.$ NOUN $: 1: / \mathrm{e} 3 \mathrm{SG}, \mathrm{eCAP} / \rightarrow \mathrm{NOUN}$ "John"] $]$

[(1 12) VP:1:/eFIN, ePRES, eAUX/ $\rightarrow$ VP: 1 SADJ $: 2$

[(1 8) VP:1:/eFIN, ePRES, eAUX/ $\rightarrow$ VP:1 PP:2

[(1 5) VP:1:/eFIN, ePRES, eAUX/ $\rightarrow$ VP:1: PP:2

$[(13)$ VP: $1: /$ eFIN, ePRES, eAUX/ $\rightarrow$

AUX"should": - eNEG VP: $1:+$ + INF-eSUBJ-eAUX

$\left[\begin{array}{ll}(1 & 2\end{array}\right)$ AUX: $1: /$ eFIN $\rightarrow->$ AUX "should" $\left.: 1\right]$

$[(23)$ VERB $: 1: /$ eFIN, eINF/ $\rightarrow$ VERB"hear" $: 1]]]$

[(3 5) PP: 1:/e3SG, eCAP, eNOMI, eCAUS/ $\rightarrow$ "from" $\mathrm{NP}: 1$ [( 45$)$ NP: $1: /$ e3SG, eCAP, eNOMI, eCAUS/

$\rightarrow$ NOUN : $1:-\mathrm{ePRO}$

$[(4 \quad 5)$ NOUN $: 1: / \mathrm{eSg}, \mathrm{eCAP} / \rightarrow$ NOUN "Mary": 1$]]]]$

[ ( 5 8) PP:1:/eDEF, eNOMI, eCAUS, e3SG/ $\rightarrow$ "about" NP: 1

$\left[\begin{array}{ll}(6 & 8\end{array}\right)$ NP $: 1 / \mathrm{eDEF}, \mathrm{eNOMI}$, eCAUS, e3SG/.

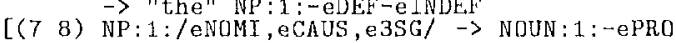

$[(7$ 8) NOUN: $1: /$ e3SG/ $\rightarrow$ NOUN"news"l] ] ] $]$

$\left[\left(\begin{array}{l}8 \\ 12\end{array}\right)\right.$ SADJ: $2: /$ eFIN, e3SG, ePRES, eSUBJ, eOBJ, eSAT $\rightarrow \rightarrow$

$[(9$ 10) NP: $1: /$ ePRD, eNOMI, e3SG, eHUM $\rightarrow$ PRON $: 1:-e P O S$

[(9 10) PRON:1:/ePRO, eNOMI, e3SG, eHUM/ $\rightarrow$ PRON"he": 1] ]

[(10 12) VP: $1:$ : eFIN, e3SG, ePRES, eOBJ, eSAT $\rightarrow$

VP"return": $1:-$ eOBJ NP"home": $2:+$ eCAUS

$[(1011)$ VP: $1: /$ eFIN, e3SG, ePRES $\rightarrow \rightarrow$ VERB $: 1:-\mathrm{ePS}$

[(10 11) VERB: 1 :/eFIN, e3SG, ePRES/

$$
\rightarrow \text { VERB "return" : 1]] }
$$

[(11 12) NP:1:/e3SG, eNOMI, eCAUS/ $\rightarrow$ NOUN: 1:-oPRO

$[(11,12)$ NOUN: $1: /$ e3SG/ $\rightarrow$ NOUN"home": 1$]] j]]$

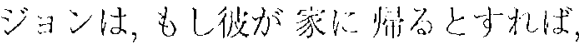

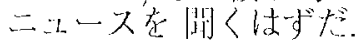

John+SUB.J, if he+SUIB.J home+GoAI, return, news + OB.J hear-tshondel

Figure 1: Sample Parsing

\section{References}

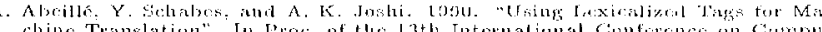

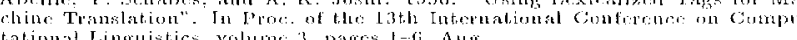

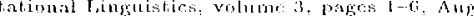

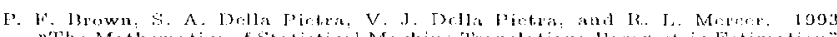

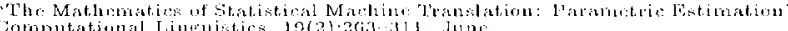

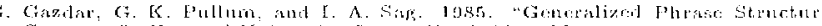

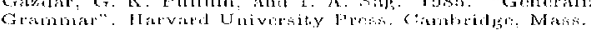

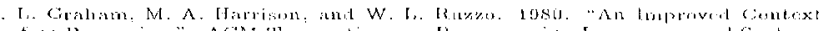

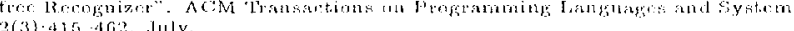
$(3): 416,462: .111 \%$

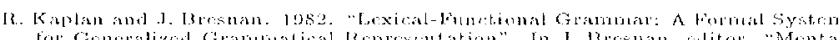

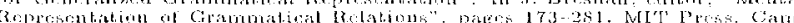
brikits: Mass.

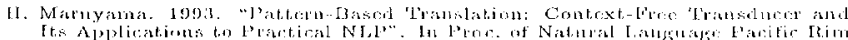

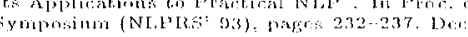

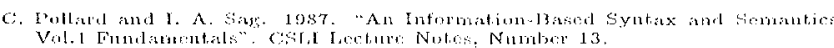

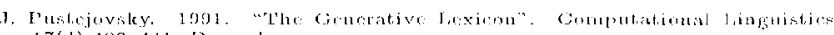

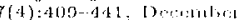

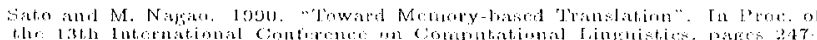
252. Hiclsinki. Aus

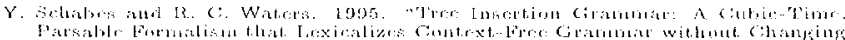

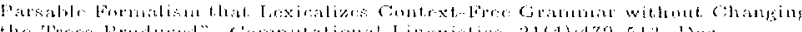

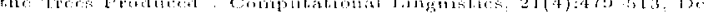

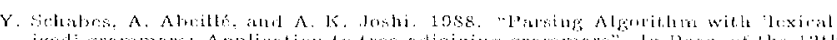

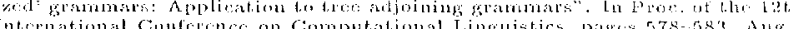

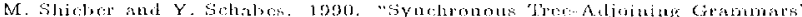

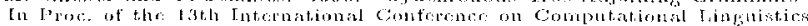

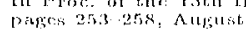

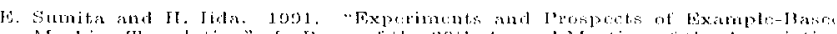

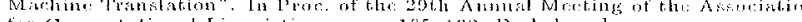

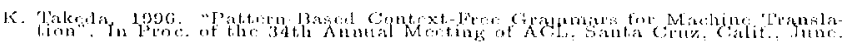

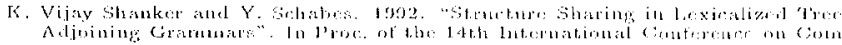

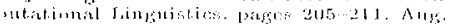

